\title{
Validación de la Escala de Prácticas Espirituales con víctimas de violencia política en Chile
}

\author{
Validation of the Spiritual Practices Scale with victims \\ of political violence in Chile
}

Héctor Arancibia ${ }^{1}$, Manuel Cárdenas ${ }^{2}$ y Cristián Ceruti $^{3}$

${ }^{1}$ Doctor en Psicología, Universidad Autónoma de Madrid. Máster en Metodología de las Ciencias del Comportamiento y de la Salud, Universidad Autónoma de Madrid. Máster en Drogodependencias, Universidad Complutense de Madrid. Psicólogo, Universidad de Chile. Profesor Titular, Instituto de Historia y Ciencias Sociales, Facultad de Humanidades y Educación, Universidad de Valparaíso. E-mail: hector.arancibia@uv.cl

${ }^{2}$ Doctor en Psicología, Universidad Autónoma de Madrid. Psicólogo, Universidad de Chile.

Profesor Titular, Facultad de Psicología, Universidad de Talca, Chile.

E-mail: jose.cardenas@utalca.cl

${ }^{3}$ Profesor Adjunto de la Facultad de Educación y Ciencias Sociales de la Universidad Andrés Bello, Chile. E-mail: cristian.ceruti@gmail.com

Universidad de Valparaíso, Chile.

\section{Resumen}

En este artículo se presenta la validación de una versión abreviada de la Escala de Prácticas Espirituales de Parsian y Dunning (2009). Para ello, se utilizó una muestra de personas adultas $(\mathrm{n}=251, \mathrm{M}=60.85$, DT $=$ 13.40) víctimas de violencia política en Chile durante la dictadura cívico militar (19731990). Para valorar la estructura de la escala se realizó un Análisis Factorial Confirmatorio (AFC), se calculó el índice de fiabilidad $(\alpha=$ .85) y se valoró la validez convergente con una serie de correlaciones bivariadas entre variables. De acuerdo a lo esperado, el AFC informa un adecuado ajuste a la estructura unifactorial y los resultados dan cuenta de la relación significativa entre las prácticas espirituales y el crecimiento postraumático y entre las prácticas espirituales y la religiosidad. La relación entre estas dos últimas es mediabaja $(r=.29)$, lo cual permite concluir que, aunque relacionadas, son constructos dife- rentes. A partir del análisis de los datos, no se puede concluir que exista relación entre las prácticas espirituales con la edad y el nivel de ingresos. En conclusión, la versión abreviada de la Escala de Prácticas Espirituales presenta adecuados niveles de fiabilidad y validez, por lo que resulta recomendable para ser utilizada en la medición de prácticas espirituales, especialmente en aquellas poblaciones que no se adscriben a una fe teísta.

Palabras clave: Escala de Prácticas Espirituales, violencia política, crecimiento postraumático, religiosidad, Chile.

\section{Abstract}

Spirituality, or what motivates the search for the sacred from particular and free positions, allows us to provide a framework from which to interpret the experience of loss and suffering, contributing in different ways to the resignification and re-authorship of people after the trauma. It is related to resilience, 
with that ability to overcome adverse events. It favors the mitigation of the negative effects of the traumatic event and fosters healthy and successful psychological development -despite the suffering caused by an external situation- by promoting the construction and redefinition of people's life purpose -one of the central components of human wellbeing-, being especially relevant in those who have been victims of trauma. Thus, spirituality can contribute to the reconfiguration of the narrative about the survived experience from practices that promote spiritual emotion such as the actions of meditating or praying, the latter considered as one of the mechanisms of self-revelation that is fundamental within the post traumatic growth process.

Spirituality and religion maintain a complex interrelation that at times makes difficult the distinction between them. However, this distinction is especially sensitive and necessary when it comes to accounting for spirituality in people who do not profess a theistic faith. This is the case of a significant part of people with left-wing ideologies -who suffered some type of violence during the military dictatorships implanted from the second half of the 20th century in Latin America. Particularly in Chile, the civic-military dictatorship (1973-1990) was especially cruel with people of left-wing ideologies, many of them not ascribed to a belief of a theistic order.

In this context, it is relevant to account for spiritual practices -differentiating them from religious beliefs- in relation to post-traumatic growth in people victims of political violence. For this objective we have proposed in this article the validation of the short version of the Parsian and Dunning (2009) Spiritual Practice Scale. For this purpose, we utilized a sample of adult people victims of political violence in Chile during the military dictatorship between 1973 and $1990(n=251$, M $=60.85, \mathrm{DT}=13.40$ ). To assess the structure of the scale, we estimated a confirmatory factor analysis (CFA). The reliability index was calculated $(\alpha=.85)$ and the convergent validity was assessed with a series of bivariate correlations between variables. As expected, the CFA reports an adequate adjustment to the unifactorial structure and the results show the significant relationship between spiritual practices and post-traumatic growth $(r=.45)$, and between spiritual practices with religiosity $(\mathrm{r}$ $=.29$ ). Being the relationship between spiritual practices and religiosity medium-low, it allows us to conclude that although they are related, they are different constructs. From the analysis of the data, it cannot be concluded that there is a relationship between spiritual practices with age and income level. In conclusion, the short version of the Spiritual Practices Scale presents adequate levels of reliability and validity, so it is recommended to be used in the measurement of spiritual practices, especially in those populations that do not adhere to a theistic faith.

Keywords: Spiritual Practices Scale, political violence, post-traumatic growth, religiosity, Chile.

\section{Introducción}

Aun cuando se asume la espiritualidad como parte esencial del ser humano (Bellamy et al., 2007; Hartrick, 2002; Urbina y Biaggio, 2000), no hay pleno consenso respecto a lo que ella significa. La dificultad para operacionalizarla (Ellison, 1983) y su enrevesada interrelación con la religión han complejizado su definición (Bergamo y White, 2016; Gall y Grant, 2005). Por un lado, la religión se entiende como un conjunto de creencias, prácticas y rituales relacionados con lo sagrado -generalmente vinculado con la divinidad y lo sobrenatural, que involucran creencias sobre espíritus, ángeles o demonios. Tiene sus raíces en una tradición establecida que surge de un grupo de personas con creencias y prácticas comunes sobre lo sagrado y se suele organizar y practicar dentro de una comunidad (Koenig, 2009). A diferencia de esta, la espiritualidad es un constructo más difícil de precisar. Una parte importante de 
las definiciones se han centrado en la noción de sentido y en los efectos que esta produce en las personas. Desde esta perspectiva, la espiritualidad podría concebirse como la raíz originaria y constitutiva de la libertad del ser humano (Frankl, 1962) que guía el quehacer de todo individuo (Narayanasamy y Owens, 2001; Ramsay y Manderson, 2011) y que, dado su componente irracional, solo es posible describirla a partir de lo que produce, de cómo "mueve el alma humana" (Otto, 2008, p. 25). Lo espiritual sería algo intocable, movilizador y que puede comprenderse de forma indirecta a partir de las acciones que promueve y de los efectos emocionales que produce, que se relacionan con la voluntad de explorar (Ramsay y Manderson, 2011, p. 170) y con la actitud activa de buscar algo que no le es conocido ni se le entrega al individuo de forma pasiva (Hill et al., 2000). Por lo general, el concepto de "espiritualidad" conlleva la sensación de interconexión con lo otro -personas, naturaleza, ente supremo, en definitiva, con algo trascendente (de Jager Meezenbroek et al., 2012; Johnstone et al., 2012; Narayanasamy y Owens, 2001; Spilka, 1993)-, pues esta sensación de interconexión es también consecuencia y causa del proceso de búsqueda (Narayanasamy y Owens, 2001).

La espiritualidad entonces, tal como fuera descripta, moviliza y surge a partir de la búsqueda de lo sagrado, categoría en la que pueden incluirse diferentes conceptos -tradicionales o no tradicionales (Zinnbauer y Pargament, 2015)-, y que no se limita a ciertos elementos específicos, pues varía dependiendo de la cultura y del momento. Lo sagrado hace alusión a aquello que puede distinguirse de lo profano por su carácter digno de veneración y respeto y, por tanto, el ser humano no puede acceder a ello sin sufrir alguna consecuencia (Durkheim, 2014), traspasando la liminalidad hacia una fase de agregación, o reagregación (Turner, 2002).

Actualmente, en occidente, la espiritualidad es una condición aceptada y valorada que se distancia de la religión-especialmente de la católica-, ya que no tiene asociado a ella el fuerte lastre de las repudiables acciones perpetradas en el pasado, que han llevado a su máxima autoridad a expresar sucesivas disculpas y peticiones de perdón durante los últimos años (i. e., en 2004 el papa Juan Pablo II -luego de siete siglos- pidió públicamente perdón por los horrores cometidos en la Santa Inquisición; en 2010 el papa Benedicto XVI pidió perdón por los abusos sexuales cometidos por sacerdotes contra menores; en 2015 el papa Francisco, durante el II Encuentro Mundial de Movimientos Populares en Bolivia, pidió perdón por los crímenes de la Iglesia católica contra los pueblos originarios durante la conquista de América y en 2018 también lo hizo por los casos de sacerdotes depredadores sexuales).

A diferencia de la religión, el uso contemporáneo de la espiritualidad está vinculado con aspectos personales, algo que la gente define por sí misma y que está en gran medida libre de las reglas, regulaciones y responsabilidades institucionales que escapan del alcance del individuo. Desde este lugar es posible entender el creciente número de personas que se definen como espirituales, pero no religiosas. Lo anterior es aún más claro al observar el caso de numerosas personas comprometidas en los procesos de lucha contra las dictaduras latinoamericanas y que poseían militancia en partidos de izquierda. Muchas de estas personas, cuando fueron sometidas a la violencia estatal, pudieron desarrollar una importante dimensión espiritual que, en algunos casos, fue un puntal importante en su proceso de recuperación con posterioridad al trauma (Cárdenas et al., 2017). Esa agregación, surgida como parte del proceso de búsqueda de la espiritualidad y/o como consecuencia de ella, es entonces parte de lo que pudiera explicar la relación entre la espiritualidad y el crecimiento postraumático.

Sin desconocer las distintas aproximaciones al concepto de espiritualidad, en este estudio se considera la definición planteada por los autores originales de la Escala de Prác- 
ticas Espirituales, quienes la definen como la búsqueda de sentido en la vida, la autorrealización y la conexión con el yo interior y el todo universal (Parsian y Duning, 2009).

\section{Violencia política, espiritualidad y crecimiento post traumático}

Durante las últimas décadas del siglo $\mathrm{XX}$, Latinoamérica atravesó por un periodo de brutales dictaduras; quedaron como testimonio de ello las personas asesinadas, desaparecidas, encarceladas y exiliadas (Muller y Bermejo, 2016). Estas dictaduras se caracterizaron por ejercer sistemáticamente violencia política, resquebrajando la salud mental de sus víctimas y de quienes las rodean. Esta forma de ejercicio de la violencia se entiende como el conjunto de acciones dirigidas a destruir o reprimir a un grupo humano -esté o no organizado- con identidad dentro de la sociedad, por su afinidad social, política, gremial, étnica, racial, religiosa, cultural o ideológica (Centro de Investigación y Educación Popular [CINEP], 2008). El impacto de estas acciones generó un trauma psicosocial, caracterizado por la vivencia o experiencia que deja una marca o herida en la persona producto de estos procesos históricos (Martín-Baró, 1988). En este contexto, la espiritualidad permite otorgar un marco desde el cual interpretar y abordar las experiencias de pérdida y sufrimiento (Seligman y Csikszentmihalyi, 2000; Shand, Cowlishaw, Brooker, Burney y Ricciardelli, 2015; Wong, 2012), la que contribuye de diferentes maneras a la reautoría de las personas luego del trauma (Pargament, Desai y McConnell, 2006; Ramsay y Manderson, 2011). Se vincula con la resiliencia al favorecer el desarrollo psicológico sano y exitoso tras el sufrimiento provocado por una situación externa (Sánchez-Teruel y Robles-Bello, 2015) y por favorecer la construcción del propósito de vida de las personas (Bergamo y White, 2016; Hill et al., 2000; Narayanasamy y Owens, 2001). La redefinición del sentido de vida forma parte de las luchas intrapersonales que se producen en quienes han sido víctimas de un trauma (Pargament et al., 2006), y es uno de los componentes centrales del bienestar humano. La espiritualidad puede contribuir a la reconfiguración de la narrativa sobre la experiencia traumática a partir de prácticas tales como la meditación o introspección que promuevan la emoción espiritual como uno de los mecanismos de autorrevelación dentro del proceso del crecimiento postraumático (Cárdenas et al., 2017; Tedeschi y Calhoun, 2004).

Aunque el aporte de la espiritualidad a los procesos de recuperación genera un creciente interés -evidenciado en el aumento de estudios sobre los efectos de la espiritualidad en situaciones de afección a la salud física y en el llamado a integrar estas dimensiones en el tratamiento $\mathrm{y}$ cuidado de personas que presentan alguna afección mental o que requieren cuidados paliativos por el envejecimiento (Agostini, 2015; Bergamo y White, 2016; Chidarikire, 2012; Cornejo y Blázquez, 2013; Ho et al., 2016; Johnstone et al., 2012; Schonfeld, Schmid y Boucher-Payne, 2016)-, la espiritualidad, al igual que la religión, no están exentas de capacidad para generar efectos detrimentales sobre el proceso de afrontamiento del trauma. Esto se hace explícito sobre todo en aquellos casos en que existen emociones de desilusión o sensaciones de abandono respecto de aquello que se considera sagrado (Bergamo y White, 2016; Gall y Grant, 2005; Pargament y Saunders, 2007), por lo que se requiere de mayor evidencia que dé cuenta de la relación entre la espiritualidad con indicadores de salud (Hill y Dik, 2012) y de crecimiento postraumático.

\section{Las medidas de la espiritualidad}

A pesar del esfuerzo de diversos autores por definir el concepto de "espiritualidad" y por diferenciarlo de la religión, una parte importante de las medidas propuestas incluyen ítems en los que aparece la idea de Dios (e. g., la Escala de Bienestar Espiritual de Palout- 
zian [2016], el Inventario de Evaluación de Espiritualidad de Hall y Edwards [2002], o la Escala de Experiencia Espiritual Cotidiana de Underwood y Teresi [2002]), con lo que pierde sentido su aplicación en personas cuya espiritualidad no se asocia con la idea de un ente superior derivado de la tradición judeocristiana, o que definitivamente no practique una espiritualidad de tipo teísta (de Jager Meezenbroek et al., 2012).

Entre los instrumentos que abordan la espiritualidad desde una perspectiva más amplia, se encuentra la Escala de Espiritualidad de Parsian y Dunning (2009), originalmente validada en EE.UU. y posteriormente traducida y validada al farsi en Irán (Aminayi, Asghari Ebrahimabad, Azadi y Soltani Shal, 2015) y al español por Díaz y otros (2012) en Colombia. Esta ha sido aplicada especialmente para medir la espiritualidad y su relación con afecciones de salud física y mental (Dunning, 2014; Mehr et al., 2014; Nikmanesh, Emamhadi y Mirabdollahi, 2013; Parsian y Dunning, 2009; Yodchai, Dunning, Savage y Hutchinson, 2017).

\section{Síntesis del estudio}

En el presente estudio se pretende validar una versión abreviada de la Escala de Prácticas Espirituales de Parsian y Dunning (2009) a partir de los resultados obtenidos por Díaz, Muñoz y Vargas (2012), por lo que, en primer lugar, se informó sobre los estadísticos descriptivos por cada ítem y sobre la distribución de los datos y, posteriormente, se evaluó la consistencia interna de la escala a través de la correlación interítem con el propósito de valorar el grado de ajuste entre los ítems (DeVon et al., 2007). Complementariamente se estimó el alfa de Cronbach para evaluar la consistencia interna de la escala. De acuerdo con los valores informados en otros contextos, para las correlaciones ítem-test se esperaban resultados mayores a .30 y para el alfa de Cronbach valores mayores a .76. Posteriormente se comprobó la validez convergente de la escala a través de correlaciones bivariadas con las variables crecimiento postraumático, religiosidad, edad e ingresos. Se esperaba una correlación media alta y significativa con el crecimiento postraumático y una correlación media-baja y significativa con la variable religiosidad. No se esperaban correlaciones significativas con las variables de nivel de ingresos y edad. Finalmente se comprobó la estructura unidimensional de la escala a través de un Análisis Factorial Confirmatorio (AFC). Dada la naturaleza ordinal de los datos, se utilizó para la estimación el procedimiento de mínimos cuadrados ponderados con estimación robusta (WLSMV) (Abad, Olea, Ponsoda, y García, 2011). Se esperaban cargas factoriales de los ítems mayores a .30 y los siguientes índices de ajuste: CFI igual o mayor a .95; TLI igual o mayor a .95; WRMR igual o menor a .90; RMSEA menor o igual a .06 (Schreiber, Nora, Stage, Barlow y King, 2006).

La validación de esta escala es especialmente relevante, ya que con ella se podrá contar con un instrumento fiable para medir prácticas espirituales en colectivos en los que se sabe o presume que no existe adscripción con alguna fe teísta. En segundo lugar, porque en Latinoamérica se ha observado una tendencia de las personas a alejarse de los tradicionales cultos y prácticas religiosas, lo cual no implica necesariamente una desvinculación de los aspectos espirituales. Este punto es aun más evidente en las personas vinculadas a la militancia política de izquierda en Chile. Se trata de personas sin adscripción a creencias religiosas, pero que en muchos casos dan cuenta de una dimensión espiritual muy desarrollada y que ha servido en sus procesos de recuperación cuando han sido víctimas de la violencia estatal. En tercer lugar, por la posibilidad de ofrecer un instrumento corto, pero al mismo tiempo robusto psicométricamente, especialmente útil cuando se hacen estudios multivariables que incluyen una gran cantidad de ítems e instrumentos. 


\section{Método}

\section{Participantes}

La muestra utilizada estuvo compuesta por 251 participantes de entre 20 y 98 años $(\mathrm{M}=$ 60.85; DT $=13.40)$, de los cuales $58.2 \%$ eran mujeres $\left(\mathrm{n}=146 ; \mathrm{M}_{\text {edad }}=59.8\right.$ años; $\left.\mathrm{DT}=12.99\right)$ y el $41.8 \%$ hombres $\left(n=105 ; M_{\text {edad }}=62.4\right.$ años; DT $=13.88)$. Los participantes compartían la condición de haber sido sobrevivientes del terrorismo de Estado ejercido en Chile entre los años 1973 y 1990 o ser sus familiares directos. Entre las formas de violencia política que los participantes declaran haber padecido, el 44.5 $\%$ fue prisionero político, el $24.1 \%$, torturado, el $37.4 \%$, familiar de un ejecutado y un 56.7 $\%$ tuvo algún familiar desaparecido. Además, un $26.8 \%$ reporta ser familiar de un prisionero político o de alguna víctima de la tortura (48.8 $\%)$. Varias de estas formas de violencia fueron experimentadas por una misma persona.

La muestra fue recolectada por medio de un procedimiento no probabilístico de carácter intencionado de tipo bola de nieve. La muestra se recogió en Chile en las ciudades de Antofagasta, Valparaíso, y Santiago. Para el comienzo del proceso de reclutamiento, dos participantes fueron seleccionados como semillas en cada ciudad, los cuales fueron refiriendo a otras personas para participar en el estudio. El cuestionario de autorreporte fue respondido por los participantes en sus hogares y fueron asesorados por encuestadores especialmente capacitados. Cada uno de los participantes firmó un consentimiento informado en el que autorizó el uso de los datos entregados, toda vez que se reguardaba la confidencialidad y el anonimato de ellos.

\section{Instrumentos}

El instrumento utilizado consideró diversas preguntas sociodemográficas y las medidas de crecimiento postraumático, de espiritualidad y de religiosidad, las cuales se detallan a continuación.

\section{Forma breve del inventario de crecimiento post traumático}

Para evaluar el crecimiento postraumático se utilizó el instrumento propuesto por Cann y otros (2010) en su versión adaptada y validada en Chile por Cárdenas, Barrientos, Ricci y Paéz (2015). Consta de diez ítems con rangos de respuesta de 1 (totalmente en desacuerdo) a 6 (totalmente de acuerdo). Puntajes más altos indican mayores niveles de crecimiento $(\alpha=.86)$.

\section{Escala de prácticas espirituales}

Para medir prácticas espirituales, se utilizó una versión modificada de la escala originalmente propuesta por Parsian y Dunning (2009) en la que se consideró el proceso de validación realizado en Colombia por Díaz y otros (2012). En esta versión se incluyeron cuatro ítems de la dimensión Prácticas espirituales ("Reflexiono para alcanzar la paz interior", "Trato de vivir en armonía con la naturaleza", "Trato de encontrar momentos para ampliar mi espiritualidad", y "Empleo el silencio para ponerme en contacto con mi yo interior"), y tres de la dimensión Necesidades espirituales ("Trato de encontrar respuesta a los misterios o dudas de la vida", "Trato de alcanzar la paz interior y la armonía", y "Busco la belleza física, espiritual y emocional en la vida") (Tabla 1). Aunque estos últimos ítems originalmente no responden a la dimensión de Prácticas espirituales, fueron incluidos a partir de la discusión de expertos disponible en la literatura (Escobar-Pérez y Cuervo-Martínez, 2008; McGartland, Berg-Weger, Tebb, Lee y Rauch, 2003) y por los resultados de la validación al castellano realizada por Díaz y otros (2012), quienes señalan que estos ítems podrían ser considerados como parte de esta dimensión. El rango de la puntuación de los ítems abarca desde 1 (totalmente en desacuerdo) hasta 6 (totalmente de acuerdo). Puntajes más altos indican mayor nivel de prácticas espirituales $(\alpha=.85)$. 


\section{Escala de religiosidad}

Para evaluar el nivel de religiosidad de los participantes, se utilizó la medida utilizada por Cárdenas y otros (2010), la cual incluye cuatro ítems: “¿Cuán fuertemente te identificas con los miembros de tu comunidad religiosa?”; “¿Cuán importante es tu religión para tu identidad?”; “Cuán a menudo piensas en términos de tus creencias religiosas?", y “¿Cuán cerca te sientes de los otros miembros de tu comunidad religiosa?", con un rango de respuesta desde 1 (nada) hasta 6 (totalmente). Puntajes más altos indican mayores niveles de religiosidad $(\alpha=.86)$.

\section{Resultados}

En la Tabla 1 se muestran los estadísticos descriptivos para cada ítem (media, desviación típica, correlación ítem-test y valor de $\alpha$ si el ítem es eliminado). El análisis de los ítems muestra que no existen casos atípicos o extremos. Los resultados evidencian el reporte de altos niveles de espiritualidad (M $=5.04, \mathrm{DT}=1.01)$. La distribución de las puntuaciones informa de una asimetría negativa $(\gamma=-1.152 ; \mathrm{ET}=.158)$ y leptocúrtica $\left(\beta_{2}\right.$ $=.158 ; \mathrm{ET}=.315)$, resultado que da cuenta de un alto nivel de prácticas espirituales de los participantes. De acuerdo a lo esperado, la correlación ítem-test varía en un rango medio a medio-alto $(r=.41$ a $r=.75)$. En cuanto a la fiabilidad de la escala $(\alpha=.85)$, la eliminación de ítems no contribuye en su incremento.

\section{Tabla 1}

Estadísticos descriptivos por item de la Escala de espiritualidad.

P1.Trato de vivir en armonía con la naturaleza.

P2. Reflexiono para alcanzar la paz interior.

P3. Trato de encontrar momentos para ampliar mi espiritualidad.

P4. Empleo el silencio para ponerme en contacto con mi Yo interior.

P5. Trato de encontrar respuesta a los misterios o dudas de la vida.

P6. Trato de alcanzar la paz interior y la armonía.

P7. Busco la belleza física, espiritual y emocional en la vida.

\begin{tabular}{|c|c|c|c|}
$\mathrm{M}$ & $\mathrm{DT}$ & $\mathrm{r}$ & $\boldsymbol{\alpha}$ \\
5.48 & .949 & .414 & .855 \\
5.10 & 1.290 & .748 & .812 \\
4.63 & 1.642 & .681 & .821 \\
4.84 & 1.574 & .586 & .836 \\
4.94 & 1.497 & .468 & .853 \\
5.25 & 1.210 & .750 & .814 \\
4.99 & 1.430 & .695 & .818
\end{tabular}

Nota: Rango de respuestas: entre 1 (totalmente en desacuerdo) y 6 (totalmente de acuerdo).

Para comprobar la validez convergente de la Escala de Prácticas Espirituales, se estimaron diversas correlaciones bivariadas entre variables (Tabla 2). De acuerdo a lo esperado, la práctica de espiritualidad presentó una correlación media-baja con la escala de religiosidad $(\mathrm{r}=.29, \mathrm{p}<.001)$. Este resultado permite concluir que, aunque ambos constructos están relacionados, se diferencian entre ellos. Las variables crecimiento postraumático y prácticas espirituales fueron las que presentaron la más alta relación entre las variables correlacionadas, lo cual permite concluir -en línea con lo esperado- que aquellas personas con mayores niveles de espiritualidad presentan mayores niveles de crecimiento postraumá- 
tico $(\mathrm{r}=.45, \mathrm{p}<.001)$. Finalmente, las prácticas espirituales no se relacionan de manera significativa con el nivel de ingresos $(\mathrm{r}=-.08$, $\mathrm{p}>.05)$ ni con la edad $(\mathrm{r}=.07, \mathrm{p}>.05)$.

Tabla 2

Correlaciones bivariadas entre varariables.

\begin{tabular}{l|l|l|l|l} 
& 1 & 2 & 3 & 4 \\
\hline 1. Espiritualidad & & & & \\
2. Religiosidad & $.291 * * *$ & & & \\
3. Crecimiento postraumático & $.454 * * *$ & $.304 * * *$ & & \\
4. Edad & $.068 *$ & $-.018 *$ & $.164 * *$ & \\
5. Ingresos & $-.079 *$ & $-.071 *$ & $-.047 *$ & $-.067 *$
\end{tabular}

${ }^{*} \mathrm{p}>.05 ; * * \mathrm{p}<.05 ; * * * \mathrm{p}<.01$

\section{Análisis factorial confirmatorio (AFC)}

Al estimar el modelo unifactorial, todos los parámetros $(\lambda)$ se encuentran adecuadamente estimados. Las magnitudes de los pesos factoriales oscilaron entre .66 y $.91 \mathrm{y}$ fueron todos significativos $(\mathrm{p}<.001)$. El estadístico chi-cuadrado fue significativo $(\mathrm{p}>.05)$. El cociente $\chi^{2} / \mathrm{gl}$ está bajo el punto de corte máximo esperado $\left(\chi^{2} / \mathrm{gl}=3.8\right)$. En cuanto a los estadísticos de ajuste, estos dan cuenta de un buen ajuste del modelo (CFI = .98; TLI = .98; WRMR $=.61)$ a excepción del error medio cuadrático de aproximación, el cual está por sobre los límites esperados $(\mathrm{RMSEA}=.11>$ .08), probablemente debido al tamaño muestral (Chen, Curran, Bollen, Kirby y Paxton, 2008; Roth, 2012).

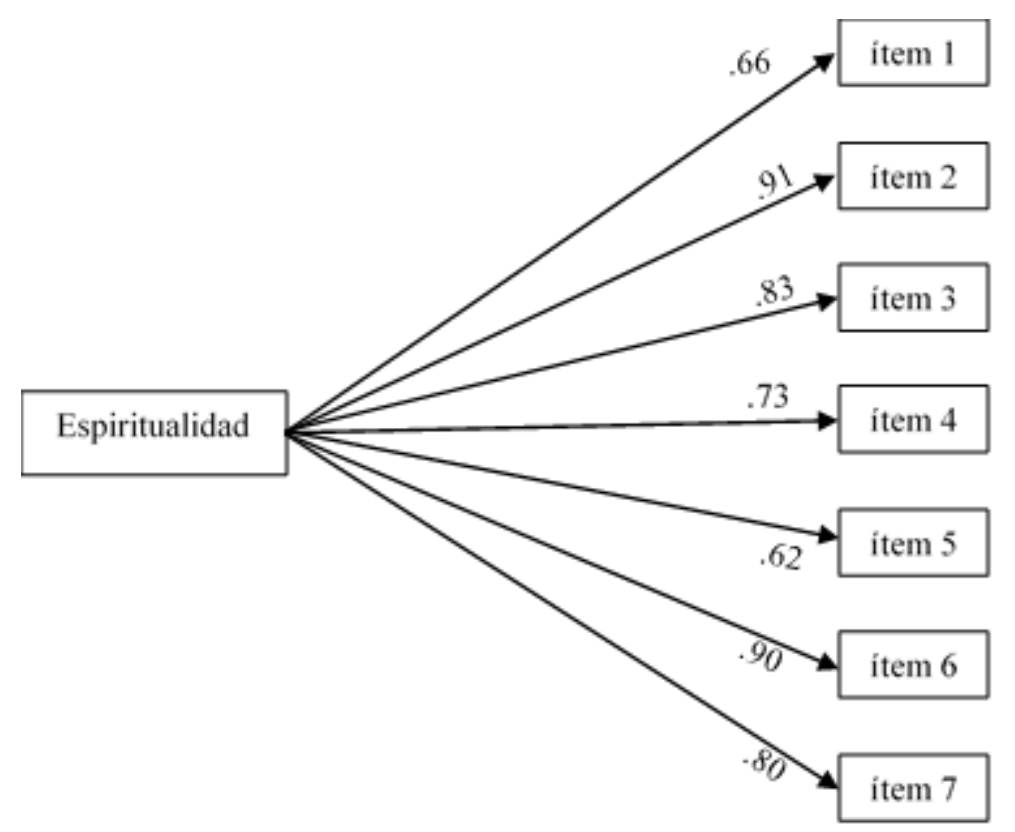

Figura 1. Modelo unifactorial de la espiritualidad con sus respectivas cargas factoriales. Notas: $(\mathrm{p}<.001) ; \chi^{2}(\mathrm{gl})=52.92(14) ; \mathrm{CFI}=.98 ; \mathrm{TLI}=.98 ; \mathrm{WRMR}=.60 ; \mathrm{RMSEA}=.11,90 \% \mathrm{IC}[.08, .14]$. 


\section{Discusión}

El propósito de esta investigación fue validar una versión abreviada de la Escala de Prácticas Espirituales con víctimas de violencia política en Chile. La distribución de los datos en la Escala de Prácticas Espirituales -basado en las medias y desviaciones estándar de cada ítem-dieron cuenta de un alto nivel de espiritualidad declarado. Estos resultados concuerdan con estudios previos que informan sobre altos niveles de espiritualidad explicitados en contextos de intimidad (Bergamo y White, 2016; Chidarikire, 2012; Schonfeld et al., 2016), tal como podría considerarse la respuesta a un instrumento de carácter autoadministrado, que fue respondido en un contexto que favorecía la introspección personal.

De acuerdo a lo esperado, las prácticas espirituales presentan una fuerte relación con el crecimiento postraumático y una relación media baja con la religiosidad. Este último resultado es relevante, ya que permite concluir que con esta escala de prácticas espirituales se está midiendo un constructo diferente al de religiosidad, razón por la cual la escala propuesta resulta especialmente útil para medir espiritualidad en aquellas personas que no tienen una creencia basada en la existencia de un dios o un ser supremo (fe teísta).

Por otra parte, la Escala de Prácticas Espirituales da cuenta de un índice de fiabilidad mayor al informado en las versiones validadas por Parsian y Dunning (2009; $\alpha=.80$ ), así como también por la versión en farsi de Aminayi y otros $(2015 ; \alpha=.77)$ y en español de Díaz y otros (2012; $\alpha=.76)$.

Los resultados de este estudio confirman la estructura unifactorial de siete ítems obtenida por Díaz y otros (2012). A modo de síntesis se puede considerar que la escala propuesta, en su versión modificada, mide adecuadamente las prácticas espirituales y que el modelo teórico que está a su base es coherente con los resultados obtenidos.

Como toda investigación, no está exenta de limitaciones. Se ha utilizado una muestra por conveniencia que, por motivos de organización y de recursos económicos, se aplicó solamente en la zona norte y central de Chile, y se excluyó la zona sur. Para futuras investigaciones se recomienda la validación de la Escala de Prácticas Espirituales en otras poblaciones y con muestras de mayor tamaño y variabilidad de rasgos que la haga más representativa de población general.

\section{Referencias}

Abad, F., Olea, J., Ponsoda, V. y García, C. (2011). Medición en ciencias sociales y de la salud. Madrid: Síntesis.

Agostini, L. (2015). Dificultades y proyecciones en la relación entre salud y espiritualidad. Veritas, 33, 143-158. https://doi.org/10.4067/ S0718-92732015000200008

Aminayi, M., Asghari Ebrahimabad, M. J., Azadi, M. y Soltani Shal, R. (2015). Reliability and validity of Farsi version of Parsian and Dunning spirituality questionnaire. Journal of Fundamentals of Mental Health, 17, 129-134.

Bellamy, C. D., Jarrett, N. C., Mowbray, O., MacFarlane, P., Mowbray, C. T. y Holter, M. C. (2007). Relevance of spirituality for people with mental illness attending consumer-centered services. Psychiatric Rehabilitation Journal, 30(4), 287-294. https://doi. org/10.2975/30.4.2007.287.294

Bergamo, D. y White, D. (2016). Frequency of Faith and Spirituality Discussion in Health Care | SpringerLink. Journal of Religion and Health, 55, 618-630. https://doi.org/10.1007/ s10943-015-0065-y

Cann, A., Calhoun, L. G., Tedeschi, R. G., Taku, K., Vishnevsky, T., Triplett, K. N. y Danhauer, S. C. (2010). A short form of the Posttraumatic Growth Inventory. Anxiety, Stress, \& Coping, 23(2), 127-137. https://doi. org/10.1080/10615800903094273

Cárdenas, M., Barrientos, J., Ricci, E. y Páez, D. (2015). Spanish Adaptation and Validation of the Posttraumatic Growth Inventory-Short Form. Violence and Victims, 30(5), 756-769. https://doi.org/10.1891/0886-6708.VV-D-13- 
00165

Cárdenas, M., Faúndez, X., Arancibia, H. y Ceruti, C. (2017). The relationship between posttraumatic growth and psychosocial variables in survivors of state terrorism and their relatives. Journal of Interpersonal Violence, 1-20. https://doi.org/10.1177/0886260517727494

Cárdenas, M., Meza, P., Lagues, K. y Yañez, S. (2010). Adaptación y validación de la Escala de Orientación a la Dominancia Social (SDO) en una muestra chilena. Universitas Psychologica, 9(1), 161-168. https://doi.org/10.11144/ Javeriana.upsy9-1.aveo

Chen, F., Curran, P. J., Bollen, K. A., Kirby, J. y Paxton, P. (2008). An Empirical Evaluation of the Use of Fixed Cutoff Points in RMSEA Test Statistic in Structural Equation Models. Sociological Methods \& Research, 36(4), 462-494. https://doi.org/10.1177/0049124108314720

Chidarikire, S. (2012). Spirituality: The neglected dimension of holistic mental health care. Advances in Mental Health, 10(3), 298-302. https://doi.org/10.5172/jamh.2012.10.3.298

CINEP (2008). Marco Conceptual Banco de Datos de Derechos Humanos y Violencia politica. Bogota.

Cornejo, M. y Blázquez, M. (2013). La convergencia de salud y espiritualidad en la sociedad postsecular. Las terapias alternativas y la constitución del ambiente holístico. Revista de Antropología Experimental, 13, 11-30.

de Jager Meezenbroek, E., Garssen, B., van den Berg, M., van Dierendonck, D., Visser, A. y Schaufeli, W. B. (2012). Measuring Spirituality as a Universal Human Experience: A Review of Spirituality Questionnaires. Journal of Religion and Health, 51(2), 336-354. https:// doi.org/10.1007/s10943-010-9376-1

DeVon, H. A., Block, M. E., MoyleWright, P., Ernst, D. M., Hayden, S. J., Lazzara, D. J., .. KostasPolston, E. (2007). A Psychometric Toolbox for Testing Validity and Reliability. Journal of Nursing Scholarship, 39(2), 155-164. https://doi.org/10.1111/j.15475069.2007.00161.x

Díaz, L., Muñoz, A. y de Vargas, D. (2012). Confiabilidad y validez del cuestionario de espiritualidad de Parsian y Dunning en versión española. Revista Latino-Americana de Enfermagem, 20(3), 1-6.

Dunning, T. (2014). Care of people with diabetes: A manual of nursing practice (4th ed). Chichester, West Sussex: John Wiley \& Sons.

Durkheim, É. (2014). Las Formas elementales de la vida religiosa. Madrid: Alianza.

Ellison, C. W. (1983). Spiritual Well-Being: Conceptualization and Measurement. Journal of Psychology and Theology, 11(4), 330-338. https://doi.org/10.1177/009164718301100406

Escobar-Pérez, J. y Cuervo-Martínez, Á. (2008). Validez de contenido y juicio de expertos: Una aproximación a su utilización. Avances en Medición, 6(1), 27-36. https://dialnet.unirioja. es/ejemplar/223670

Frankl, V. (1962). El hombre en busca de sentido. Barcelona: Herder.

Gall, T. L. y Grant, K. (2005). Spiritual Disposition and Understanding Illness. Pastoral Psychology, 53(6), 515-533. https://doi. org/10.1007/s11089-005-4818-y

Hall, T. W. y Edwards, K. J. (2002). The Spiritual Assessment Inventory: A Theistic Model and Measure for Assessing Spiritual Development. Journal for the Scientific Study of Religion, 41(2), 341-357. https://doi.org/10.1111/14685906.00121

Hartrick, G. A. (2002). Beyond polarities of knowledge: The pragmatics of faith. Nursing Philosophy, 3(1), 27-34. https://doi.org/10.1046/ j.1466-769X.2002.00079.x

Hill, P. y Dik, B. (Eds.). (2012). Psychology of religion and workplace spirituality. Charlotte, N.C: Information Age Pub., Inc.

Hill, P., Pargament, K., Hood, R. W., McCullough, J., Michael E., Swyers, J. P., Larson, D. B. y Zinnbauer, B. J. (2000). Conceptualizing Religion and Spirituality: Points of Commonality, Points of Departure. Journal for the Theory of Social Behaviour, 30(1), 51-77. https://doi. org/10.1111/1468-5914.00119

Ho, R. T. H., Chan, C. K. P., Lo, P. H. Y., Wong, P. H., Chan, C. L. W., Leung, P. P. Y. y Chen, E. Y. H. (2016). Understandings of spirituality and its role in illness recovery in persons with 
schizophrenia and mental-health professionals: A qualitative study. BMC Psychiatry, 16(1), 86. https://doi.org/10.1186/s12888-016-0796-7

Johnstone, B., Yoon, D. P., Cohen, D., Schopp, L. H., McCormack, G., Campbell, J. y Smith, M. (2012). Relationships Among Spirituality, Religious Practices, Personality Factors, and Health for Five Different Faith Traditions. Journal of Religion and Health, 51(4), 10171041. https://doi.org/10.1007/s10943-0129615-8

Koenig, H. G. (2009). Research on Religion, Spirituality, and Mental Health:AReview. The Canadian Journal of Psychiatry, 54(5), 283-291. https://doi.org/10.1177/070674370905400502

Martín-Baró, I. (1988). La violencia política y la guerra como causas del trauma psicosocial en El Salvador. Revista de Psicología de El Salvador, 7(28), 123-141.

McGartland, D., Berg-Weger, M., Tebb, S., Lee, S. y Rauch, S. (2003). Objectifying content validity: Conducting a content validity study in social work research. Social Work Research, 27(2), 94-104. https://doi.org/10.1093/ swr/27.2.94

Mehr, S. E., Pirastehmotlagh, A., Aliabad, T. A., Ansari, Y., Rozeyan, A., Derakhsh, A. y Mohamadi, F. (2014, octubre 1). The relationship between variables of spirituality, attitude toward the disease and suffering in aids cases. Recuperado 22 de agosto de 2019, de Advances in Environmental Biology website: https:// link.galegroup.com/apps/doc/A417895265/ AONE? $\mathrm{sid}=1 \mathrm{~ms}$

Muller, F. y Bermejo, F. (2016). Los temas de la memoria colectiva del golpe de estado de 1976 en Argentina en función de la ideología y las generaciones. Interdisciplinaria, 33(1), 41-63. https://doi.org/10.16888/interd.2016.33.1.3

Narayanasamy, A. y Owens, J. (2001). A critical incident study of nurses' responses to the spiritual needs of their patients. Journal of Advanced Nursing, 33(4), 446-455. https://doi. org/10.1046/j.1365-2648.2001.01690.x

Nikmanesh, Z., Emamhadi, M. y Mirabdollahi, N. (2013). Predictions of post-traumatic growth according to spirituality, social support and positive affection in deferent age groups with breast cancer. Journal of Research and Health, 3(2), 355-362.

Otto, R. (2008). Lo sagrado. Buenos Aires: Claridad.

Paloutzian, R. F. (2016). The Spiritual Well-Being Scale: Portuguese Translation and Suggestions for Use. Horizonte, 14(41), 76-88. https://doi. org/10.5752/P.2175-5841.2016v14n41p76

Pargament, K. I., Desai, K. M. y McConnell, K. M. (2006). Spirituality: A Pathway to Posttraumatic Growth or Decline? En Handbook of posttraumatic growth: Research \& practice (pp. 121-137). Mahwah, NJ, US: Lawrence Erlbaum Associates Publishers.

Pargament, K. I. y Saunders, S. M. (2007). Introduction to the special issue on spirituality and psychotherapy. Journal of Clinical Psychology, 63(10), 903-907. https://doi.org/10.1002/ jclp. 20405

Parsian, N. y Dunning, T. (2009). Developing and validating a questionnaire to measure spirituality: A psychometric process. Global Journal of Health Science, 1(1), 2-11.

Ramsay, T. y Manderson, L. (2011). Resilience, Spirituality and Posttraumatic Growth: Reshaping the Effects of Climate Change. En I. Weissbecker (Ed.), Climate Change and Human Well-Being: Global Challenges and Opportunities (pp. 165-184). https://doi. org/10.1007/978-1-4419-9742-5_9

Roth, E. (2012). Análisis Multivariado en la Investigación Psicológica: Modelado Predictivo y Causal con SPSS y AMOS. La Paz: Universidad Católica Bolivariana.

Sánchez-Teruel, D. y Robles-Bello, M. A. (2015). Escala de resiliencia 14 ítems (RS-14): Propiedades psicométricas de la versión en español. Revista Iberoamericana de Diagnóstico y Evaluación-e Avaliação Psicológica, 2(40), 103-113.

Schonfeld, T. L., Schmid, K. K. y BoucherPayne, D. (2016). Incorporating Spirituality into Health Sciences Education. Journal of Religion and Health, 55(1), 85-96. https://doi. org/10.1007/s10943-014-9972-6

Schreiber, J. B., Nora, A., Stage, F. K., Barlow, 
E. A. y King, J. (2006). Reporting Structural Equation Modeling and Confirmatory Factor Analysis Results: A Review. The Journal of Educational Research, 99(6), 323-338. https:// doi.org/10.3200/JOER.99.6.323-338

Seligman, M. E. y Csikszentmihalyi, M. (2000). Positive psychology: An introduction. American Psychologist, 55(1), 5-14. https://doi. org/10.1037//0003-066X.55.1.5

Shand, L. K., Cowlishaw, S., Brooker, J. E., Burney, S. y Ricciardelli, L. A. (2015). Correlates of post-traumatic stress symptoms and growth in cancer patients: A systematic review and meta-analysis. Psycho-Oncology, 24(6), 624-634. https://doi.org/10.1002/ pon. 3719

Spilka, B. (1993). Spirituality: Problems and directions in operationalizing a fuzzy concept. Presentado en Toronto, Ontario. Toronto, Ontario: American Psychological Association.

Tedeschi, R. G. y Calhoun, L. G. (2004). Posttraumatic Growth: Conceptual Foundations and Empirical Evidence. Psychological Inquiry, 15(1), 1-18. https://doi.org/10.1207/ s15327965pli1501_01

Turner, V. (2002). The forest of symbols: Aspects of Ndembu ritual. Ithaca, NY: Cornell Univer- sity Press.

Underwood, L. G. y Teresi, J. A. (2002). The daily spiritual experience scale: Development, theoretical description, reliability, exploratory factor analysis, and preliminary construct validity using health-related data. Annals of Behavioral Medicine, 24(1), 22-33. https://doi. org/10.1207/S15324796ABM2401_04

Urbina, C. y Biaggio, A. (2000). Adaptación de un Cuestionario sobre Grado de Fe, para Brasil y Chile. Revista iberoamericana de diagnóstico y evaluación psicológica, 10(2), 94-100.

Wong, P. T. P. (Ed.). (2012). The human quest for meaning: Theories, research, and applications (2nd ed). New York: Routledge.

Yodchai, K., Dunning, T., Savage, S. y Hutchinson, A. M. (2017). The role of religion and spirituality in coping with kidney disease and haemodialysis in Thailand. Scandinavian Journal of Caring Sciences, 31(2), 359-367. https://doi. org/10.1111/scs.12355

Zinnbauer, B. y Pargament, K. (2015). Religiousness and Spirituality. En R. Paolutzian y C. Park (Eds.), Handbook of the psychology of religion and spirituality. https://doi. org/10.4324/9781315662732-8

Recibido: 7 de junio de 2020 Aceptado: 6 de octubre de 2021 